\title{
Managing Service Operations based on Customer Preferences
}

\author{
Rohit Verma \\ DePaul University, Chicago, Illinois, USA \\ Gary M. Thompson \\ Cornell University, Ithaca, New York, USA
}

\begin{abstract}
This article presents the results of a study using discrete choice analysis (DCA) in the dine-in pizza industry. DCA offers an effective approach for incorporating customer preferences into operating decisions in service businesses. Our results show how customers tradeoff among several determinant attributes (e.g. price, waiting time, quality) when choosing a dine-in pizza restaurant. The article also offers evidence that managers' perceptions of customer choice patterns are not the same as customers' actual choice patterns for the businesses we examined. Finally, we show how our results can be easily incorporated into a decision support system for structuring service operations according to customer preferences.
\end{abstract}

\section{Introduction}

As the field of Service Operations Management moves beyond the "exploratory" research stage, a number of scholars have emphasized that an integration of operations and marketing perspectives are essential for design and management of high performing services (Chase, 1996). For example, Griffen and Hauser (1993) emphasize the usefulness of integrating the voice of the customer into service businesses. Lovelock (1992) suggests an integrated approach to service management using a combination of marketing, operations and human resources perspectives. Davidow and Uttal (1989) suggest using market segmentation analysis to determine service strategies for different segments of customers and better match supply and demand. Similarly, Heskett's (1987) "strategic service vision" consists of the identifying of a target market segment, developing a service concept to address targeted customers' needs, codifying an operating strategy to support the service concept, and designing a service delivery system to support the operating strategy. Hart (1988) advocates using unconditional service guarantees and suggests that they offer a very powerful strategy for service business. An unconditional service guarantee pushes the entire company to focus on the customers' definition of good service and not on any executive's assumptions.

The above publications describe the multi-disciplinary nature of service operations and provide directions for effective management. At the same time another series of articles 
presents a variety of service classification schemes with the objective of identifying better service designs. For example, Lovelock (1983) classified services in five different two-by-two matrices and examined how the specific nature of services in a particular class affects operations and marketing. Chase (1981) proposed that if there is less direct customer contact in the service system, then the service system is more likely to operate at its peak efficiency. Mersha (1990) proposed a broadened definition of customer contact and extended Chase's customer contact model. Also building on the customer contact approach, Schmenner (1986) proposed the Service Process Matrix (SPM), based on three characteristics of service delivery systems: customer contact; service customization; and labor intensity. Wemmerlov (1990) proposed a taxonomy of service processes that included degree of customer contact, complexity and divergence, while Kellog and Nie (1995) proposed a two-dimensional matrix which connected the characteristics of service products with service processes.

The motivation for our work stems from two streams of research. First, a number of articles have argued that service research has moved beyond the primary classification stage and therefore now it is necessary to validate the generally accepted concepts/frameworks and develop specific methodologies for service design and process improvement (Chase, 1996; Flynn et al., 1990; Meredith et al., 1989; Swamidass, 1991). The second motivation for our work comes from a series of recent articles which argue that in order to effectively compete in a competitive marketplace, service companies must develop a coherent operations strategy (Vickery et al., 1993). We will demonstrate the linkages between competitive priorities (product and service quality, cost, delivery and flexibility) and customer choice patterns for one specific industry.

This article presents an econometric procedure known as discrete choice analysis (DCA) which can be used to effectively integrate market/customer preference information into operating decisions in service factories. The service typologies described earlier provide conceptual frameworks for analyzing the differences between service industries; however, they do not suggest how a particular company can position itself within its industry for higher profit and/or market share. The approach presented in this article can be used to align the productservice package offerings of a particular company within a given industry and market structure.

Specifically, this paper demonstrates an approach for identifying:

(1) customer preferences based on quality, cost, delivery or flexibility attributes of productservice packages offered in the marketplace;

(2) managers' perceptions of customer product-service choice patterns; and

(3) the gap between customer preferences and managers' perceptions.

In the past, DCA has been successfully used for a variety of applications in marketing, consumer research, transportation, recreation and leisure research, sociology and other social sciences (for example: Ben-Akiva and Lerman, 1991; McFadden, 1986; Louviere and Timmermans, 1990; 
Verma and Thompson, 1996). Please refer to a recent paper by Verma et al. (1999) for guidelines for conducting DCA experiments for the service operations.

The empirical data presented in this study was collected from the managers and customers of three dine-in pizza restaurants located close to a large state university (approx. student population 25,000) in the United States of America. The same corporation owns these three restaurants and the majority of their customers are the students and staff members of the university. The results presented in this article show first, how the customers of the pizza shops tradeoff among various determinant attributes (quality, cost, delivery, and flexibility), and what managers think their customers need. We highlight key differences (gaps) between the choice patterns of the two samples (managers vs. customers) and draw implications for the design and operation of the service facilities.

Although past research in service management has studied tradeoffs among different service attributes, none of the studies have used methods similar to DCA to identify customer choices and/or the gaps between customer choices and managers' perceptions. For example, Davis (1991) studied the tradeoff between customer waiting time and operations efficiency. Similarly Lindsley et al. (1991) studied the tradeoff between time and product variety in the book distribution industry and recommended that managers should be aware of relative values of time and variety in their distribution strategy for better service management. Even though these articles provide valuable information related to service operations management, they only study two (or three) service attributes. Effectively incorporating customer preferences into service operations management requires that one identify the relative importance of all (or most) of the attributes customers consider relevant.

The rest of the article is organized in four sections. First, we present a brief overview of the DCA and describe the research design; second, we present the result of the empirical study conducted in the dine-in pizza; and third, we discuss the implications of using research approaches similar to DCA in service operations management.

\section{Research Approach}

In order to meet customer demand in a dynamically changing competitive environment, it is important to listen carefully to the voice of the customer (Griffin and Hauser, 1993). Past research shows that customers choose from a set of alternatives, the product/service that has the highest utility for them (McFadden, 1986; Ben-Akiva and Lerman, 1991; Louviere, 1988; Verma et al., 1999). After acquiring information and learning about the alternatives, consumers define a set of determinant attributes to use, and then compare products in a particular product/service class (Verma et al., 1999). After consumers form impressions of the positions of various alternatives on the determinant attributes, they make value judgements and combine information to form overall impressions of the alternatives. In order to do so, they have to make tradeoffs among different product/service attributes (Anderson, 1981, 1982). 
Discrete choice analysis (DCA) identifies the coefficients for different attributes based on the decision maker's response to experimentally designed profiles of possible alternatives and models the evaluation process described above. DCA involves designing several experimental profiles (alternatives) of decision situations (e.g. profiles of quick service restaurants with different food quality, cost, delivery time) and asking the decision maker(s) to choose an alternative from a set of possible choices. A typical discrete-choice experiment simultaneously shows two or more alternatives to the decision maker and asks him/her to choose one. This choice making process is repeated several times. Next, multinomial logit (MNL) (or more complex econometric) model is used to identify the weights and statistical significance of the attributes. The MNL model assumes that the probability of selecting an alternative depends on the decision maker's perceptions of the relative "attractiveness" or "utilities" of the alternatives (Louviere, 1988).

Designing and conducting discrete choice experiments involves the following steps: identifying the attributes; specifying attribute levels; designing an experiment; presenting sets of alternatives to respondents; and estimating a model. For the sake of clarity, the next subsection briefly summarizes the above DCA steps. For a detailed review of DCA methodology, please refer to a recent review article by Verma et al. (1999).

\section{Discrete Choice Analysis: A Review}

A discrete choice experiment requires one to identify the determinant attributes used by decision makers to evaluate alternatives. Qualitative surveys, interviews, case studies, and/or focus groups and a review of academic and practitioner literature can be used to identify a set of relevant attributes (Verma et al., 1999). The final number of attributes selected often reflects a balance between completeness and complexity (Louviere et al., 1995).

The researcher's next task is to determine the range of possible values for each of the identified attributes. Chosen attribute ranges can either span the actual values observed in the marketplace and/or represent values expected to be observed during the planning horizon. Response reliability may suffer if unrealistic attribute ranges are used (Louviere and Timmermanns, 1990). Next the attribute range is categorized into two or more levels for the purpose of experimental design. Two levels for each attribute (e.g. the lowest and highest possible values of an attribute) are sufficient to estimate linear effects of attributes on choice, but one needs more than two levels to estimate a nonlinear effect of the attribute on choice (Hagerty, 1986).

After the identification of relevant attributes and their levels, experimental design procedures are used to generate descriptions (profiles) of hypothetical (but possible) productservice alternatives. Factorial experimental designs allow one to create descriptions of choice alternatives such that all statistical effects of attributes can be estimated independently (Bishop et al., 1975; Hahn and Shapiro, 1966; Louviere, 1988; McLean and Anderson, 1984). In practice, however, complete factorial designs rarely can be used because they generate large numbers of 
combinations. Instead, fractional factorial designs are used to construct a limited number of profiles. Fractional factorial designs assume that one or more interactions (or the linear combinations of attribute effects) among the attributes are not statistically significant. Next, the experimentally generated profiles must be grouped together in some way to generate choice sets. Depending on the number of attributes, their levels, and number of alternatives per choice set, anywhere from eight to $100+$ choice sets are created.

Next, the survey instrument containing the discrete choice sets are presented to the selected respondents. A wide range of media can be used to describe hypothetical choice alternatives generated by the experimental design. For example, one may use sentences, short phrases, or paragraphs; pictures, drawing, photographs, or computer images; models; or any combination of written, visual or other sensory representation. The choice of presentation media is situational and the best medium for one study might not be appropriate for another.

The final aspect of designing discrete choice experiments involves the format of the choice task. Most published DCA studies present two or more alternatives at the time to the decision makers and ask them to choose one (or none) of them (Louviere and Timmermans, 1990; Verma and Thompson, 1996). It is also possible to show only one experimental profile at a time to decision makers and observe yes/no (accept/reject) responses.

After generating choice sets and formatting the choice task, empirical data are collected from multiple respondents. Naturally, respondents should be chosen to be representative of the population of interest, otherwise the results will not generalize to the whole population. Depending on the size and complexity of the experiment, subjects can be asked to respond to all choice sets or one can divide the choice sets into two or more statistically equivalent subsets and randomly assign subjects such that each responds to one subset of the choice sets.

\section{Choice Model Estimation}

Empirical data collected from respondents to discrete choice experiments are categorical because respondents choose only one alternative (normally coded as 1; all other alternatives coded as 0 ) in each choice set. Thus, one cannot estimate discrete choice models for single individuals because there are too few observations per individual to satisfy the asymptotic conditions needed to obtain consistent and efficient parameter estimates (BenAkiva and Lerman, 1991). Satisfaction of asymptotic properties ordinarily requires large numbers of observations of discrete choices (at least six respondents). Therefore responses from various subjects typically are aggregated and used to estimate choice models, although various segmentation schemes can be used to deal with respondent heterogeneity. The most common form of the econometric model based on discrete choice analysis is known as the multinomial logit (MNL) model (Ben Akiva and Lerman, 1991; Verma et al., 1999), which was also used in our study. The MNL model is expressed as 


$$
P_{i j}=\frac{e^{\mu V_{i j}}}{\sum_{k=1}^{K} e^{n \mu V_{k j}}}
$$

where: $P_{i j}$ represents the probability of selecting alternative $i$ from the $j$ th set containing $K$ alternatives, and $V_{i j}$ represents the systematic utility of alternative $i$ in choice set $j$.

The MNL model assumes that the errors are independent and identically distributed according to a Gumbel distribution with a scale parameter $\mu$. Representing a product or service as a bundle of its attributes, and by assuming an additive utility function, an alternative's utility can be calculated as:

$$
V_{i j}=\sum_{l=1}^{L} \beta_{l} x_{i j l}
$$

where $x_{i j l}$ is the level of attribute $l$ of alternative $i$ in choice set $j, \beta_{l}$ is the relative utility weight (part-worth utility) associated with attribute $l$, and $L$ is the total number of attributes.

Although a number of approaches can be used to estimate MNL parameters, maximum likelihood is preferred (Ben-Akiva and Lerman, 1991). The likelihood function for $M$ subjects can be represented as

$$
L=\prod_{m=1}^{M} \prod_{l=1}^{L} \prod_{j=1}^{J} P_{i j}^{Y_{i j m}}
$$

where

$$
Y_{i j m}=\left\{\begin{array}{l}
1, \\
0,
\end{array} \text { if subject } m \text { chooses alternative } i \text { in choice set } j\right. \text { oterhwise. }
$$

Several likelihood ratio tests (similar to the F-test in ordinary least square regression) can be used to test the statistical significance of the estimated MNL choice models. A log-likelihood ratio test is based on the differences between the natural logarithm of the likelihood function (equation (3)) under two conditions. First the likelihood ratio is calculated assuming there are equal probabilities of choosing each alternatives in a choice set (or by assuming that all $\beta_{l}$ parameters equal zero). This natural logarithm of the likelihood (loglikelihood) value is represented as $\mathrm{LL}(0)$. Next, the likelihood ratio is calculated again, assuming the estimated $\beta_{l}$ parameters. This log-likelihood value is called LL(B). Then, the log-likelihood ratio test is defined as

$$
-2[L L(0)-L L(B)]
$$

and is $\chi^{2}$ distributed with the degrees of freedom equal to the number of $\beta$ parameters. McFadden's $\rho^{2}$ and adjusted McFadden's $\rho^{2}$ measures (similar to $R^{2}$ and adjusted $R^{2}$ in OLS regression) are defined in the following manner:

$$
\rho^{2}=1-[L L(B) / L L(0)]
$$




$$
\text { Adjusted } \rho^{2}=1-\left[\left(L L(B)-\text { number of } b_{l} \text { parameter }\right) / L L(0)\right]
$$

In order to investigate if two choice models are statistically similar to each other (e.g. customer vs. manager models) a $\chi^{2}$ test developed by Swait and Louviere (1993) is used. This procedure first rescales Gumbel scale parameters (in equation 1 ) and then compares the models using the following $\chi^{2}$ statistic with $L+1$ degrees of freedom ( $L$ is the number of attributes):

$$
-2\left[L L_{\mu}-\left(L L_{1}+L L_{2}\right)\right]
$$

Where $L L_{1}$ and $L L_{2}$ are the log-likelihood values of the two multinomial logit models without any rescaling, and $L L_{\mu}$ is the log-likelihood value for the joint model with a rescaling parameter $\mu$.

To identify the relevant attributes for the dine-in-pizza restaurants located close to the university campus, we collected qualitative information from 15 randomly selected undergraduate and graduate students. According to Griffin and Hauser (1993) between ten to 20 subjects are enough to identify the majority of attributes used by the customers in a given market segment in choosing a product/service. We conducted short interviews and asked the selected students to list the relevant variables for dine-in pizza restaurants. Based on their responses we selected the 15 attributes of dine-in pizza restaurants to be used in the further analysis. These attributes were considered to be important by at least three of the 15 respondents.

Past research in operations management and marketing suggests that customers choose products and services based on product quality, service quality, cost, delivery and flexibility attributes (Hayes and Wheelwright, 1984; Anderson et al., 1989). Table I presents the theoretical constructs (product quality, service quality, cost, delivery and flexibility) behind the 15 attributes we used. We appreciate the argument that product quality, service quality and flexibility are multidimensional in nature and therefore several other variables might be necessary to adequately represent these theoretical constructs. However, the objective of this research is not to test theoretical constructs but to understand the choice behavior of customers in one market segment and to position service operations accordingly. Therefore, we only included the variables mentioned by 20 per cent or more (three or more out of 15) respondents. Additionally, since the research methodology (discrete choice analysis) is based on a factorial experimental design, including all possible attributes will increase the dimensionality of the study considerably. Louviere and Timmermans (1990) recommend such an approach and suggest that one should re-combine or re-express attributes to keep the set of attributes as nonredundant and as small as possible to make an experiment manageable yet realistic.

Next, we used experimental design software, CONSURV (Intelligent Marketing Systems, 1992 ) to generate 32 orthogonal fractional factorial profiles of dine-in pizza companies. These profiles contained one of the two levels of the attributes presented in Table II (since the price of 


\begin{tabular}{ll}
\hline Construct & Attributes \\
\hline Cost & Price of a small pizza \\
& Price of a medium pizza \\
& Price of a large pizza \\
Welivery & Waiting time (to seat) \\
& Waiting time (to order) \\
& Waiting time (to get the food - after ordering) \\
Flexibility & Types of pizza crust available \\
& Types of pizza toppings available \\
Product quality & Amount of toppings on the pizza \\
& Amount of cheese on the pizza \\
& Pizza crust uniformity \\
& Pizza taste consistency \\
Service quality & Aesthetics of the restaurant \\
& Reliability of service \\
& Will handle unique customer demands? \\
& Knowledgeability and skills of the employees \\
& Unconditional service guarantee offered?
\end{tabular}

Table I. Attributes of dine-in pizza establishments selected for experimental design

three types of pizza sizes are multi-collinear in nature, we included only one of the price variables in the experimental design). The price of the medium pizza was assumed to be \$4 less than that of the large pizza and the price of the small pizza was assumed to be $\$ 4$ less than that of the medium pizza. The 32-profile design we used can estimate all the main effects of the variables represented in Table II. We used two levels for each attribute in experimental design. If we had used four levels for each variable, the dimensionality of the experiment would have increased from $2^{15}$ to $4^{15}$ (i.e. by a factor of $2^{15}$, or 32,768 times).

Next, we grouped several experimentally generated profiles together to generate discrete choice sets. To our knowledge, there is no agreement on the "best" method for generating the choice sets (see, for example, the texts by Ben-Akiva and Lerman, 1991; Louviere, 1988). If only two attributes are used for every variable then every profile can be combined with its "foldover" profile to generate an efficient choice set with two alternatives. A foldover design contains the opposite levels of every attribute for a given profile. We used this procedure to develop choice sets for the pizza study. Table III presents a sample choice set. Table III shows that Restaurant \#2's attribute levels are opposite to those of Restaurant \#1.

Since the primary customers of the three dine-in pizza restaurants were the students of the university, we randomly selected 100 students as respondents. Approximately two-thirds of the student sample consisted of undergraduate students, with the remaining students enrolled in various graduate programs. As the subjects were selected from various parts of the university campus, the sample represents students in a variety of academic disciplines. Six students did not complete the survey instrument and five others mentioned that they do not like pizza, resulting in a final sample size of 89. Additionally we contacted the managers of the three pizza restaurants and collected their responses. Each subject (customers and managers) responded to 32 discrete sets (similar to Table III). The student subjects were asked to chose their preferred restaurant and the managers were asked to predict the choice patterns of student customers. 


\begin{tabular}{|c|c|c|}
\hline Attributes & $\begin{array}{l}\text { Level \#1 } \\
\text { (experimental design } \\
\text { code }=-1 \text { ) }\end{array}$ & $\begin{array}{l}\text { Level \#2 } \\
\text { (experimental design } \\
\text { code }=+1 \text { ) }\end{array}$ \\
\hline Price of a small pizza & $\$ 3$ & $\$ 8$ \\
\hline Price of a medium pizza & $\$ 7$ & $\$ 12$ \\
\hline Price of a large pizza & $\$ 11$ & $\$ 16$ \\
\hline Waiting time (to seat) & 5 mins & 20 mins \\
\hline Waiting time (to order) & 5 mins & 20 mins \\
\hline $\begin{array}{l}\text { Waiting time (to get the food - } \\
\text { after ordering) }\end{array}$ & 15 mins & 45 mins \\
\hline Types of pizza crust available & One type & Three different types \\
\hline Types of pizza toppings available & Traditional & Traditional + gourmet \\
\hline Amount of toppings on the pizza & Little & Lots \\
\hline Amount of cheese on the pizza & Little & Lots \\
\hline Pizza crust uniformity & Always consistent & Varies a lot \\
\hline Pizza taste consistency & Always consistent & Varies a lot \\
\hline Aesthetics of the restaurant & $\begin{array}{l}\text { Looks like a fast-food } \\
\text { restaurant }\end{array}$ & $\begin{array}{l}\text { Looks like an Italian } \\
\text { restaurant }\end{array}$ \\
\hline Reliability of service & $\begin{array}{l}\text { Arrives } 15 \text { mins. later } \\
\text { than told }\end{array}$ & Arrives when promised \\
\hline $\begin{array}{l}\text { Will handle unique customer } \\
\text { demands? }\end{array}$ & Yes & Yes \\
\hline $\begin{array}{l}\text { Knowledgeability and skills of the } \\
\text { employees }\end{array}$ & $\begin{array}{l}\text { Very knowledgeable, } \\
\text { polite and friendly }\end{array}$ & $\begin{array}{l}\text { Not very knowledgeable, } \\
\text { polite and friendly }\end{array}$ \\
\hline $\begin{array}{l}\text { Unconditional service guarantee } \\
\text { offered? }\end{array}$ & Yes & Yes \\
\hline
\end{tabular}

Table II. Dine-in pizza restaurant: attributes and their levels

\section{Results and Analysis}

\section{Consumer choice model}

Table IV shows the estimated multinomial logit model (MNL) for the sample of 89 customers (or 2,848 responses). A negative $\beta$ means that the probability of a customer selecting a particular restaurant will increase if the level of that particular attribute is reduced. For example, based on the MNL model presented in Table IV, the market share of a particular pizza shop will increase if the price or waiting times are reduced. Similarly, a positive $\beta$ means that increasing the level of a particular attribute will increase the market share of a particular restaurant. For example, a pizza restaurant can increase its market share by offering more pizza crust options or more types of toppings (both have positive $\beta$ in Table IV). Additionally, we standardized the design code (mean $=0$; standard deviation $=1$ ) for all the variables presented in Table IV and so the absolute value of a $\beta$ parameter represents an attribute's relative importance for the customer. Therefore according to Table IV, reliability of service and amount of toppings on the pizza are the two most important attributes for the customers.

Table IV also contains statistical information related to the MNL model for the customers. The log-likelihood test (equation (4)) tests the statistical significance of the overall model. The estimated log-likelihood ratio was found to be 985.34 , which is $\chi^{2}$ distributed with 16 degrees of freedom ( 15 variables and one intercept) and is statistically significant at the 5 per cent level. The McFadden's $\rho^{2}$ (similar to $R^{2}$ in Ordinary Least Square Regression) was found to be 0.74 which means that approximately 74 per cent of the variation in the dependent variable (customer choice) was captured by the estimated MNL model. Table IV also presents 


\begin{tabular}{|c|c|c|}
\hline Variables & Restaurant \#1 & Restaurant \#2 \\
\hline Price of a small pizza & $\$ 3$ & $\$ 8$ \\
\hline Price of a medium pizza & $\$ 7$ & $\$ 12$ \\
\hline Price of a large pizza & $\$ 11$ & $\$ 16$ \\
\hline Waiting time (to seat) & 20 mins & 5 mins \\
\hline Waiting time (to order) & 5 mins & 20 mins \\
\hline $\begin{array}{l}\text { Waiting time (to get the food - } \\
\text { after ordering) }\end{array}$ & 45 mins & 15 mins \\
\hline Types of pizza crust available & One type & Three different types \\
\hline Types of pizza toppings available & Traditional + gourmet & Traditional only \\
\hline Amount of toppings on the pizza & Little & Lots \\
\hline Amount of cheese on the pizza & Lots & Little \\
\hline Pizza crust uniformity & Varies a lot & Always consistent \\
\hline Pizza taste consistency & always consistent & varies a lot \\
\hline Aesthetics of the restaurant & $\begin{array}{l}\text { Looks like a fast-food } \\
\text { restaurant }\end{array}$ & $\begin{array}{l}\text { Looks like an Italian } \\
\text { restaurant }\end{array}$ \\
\hline Reliability of service & Arrives when promised & $\begin{array}{l}\text { Arrives } 15 \text { mins later } \\
\text { than told }\end{array}$ \\
\hline Will handle unique customer demands? & Yes & No \\
\hline $\begin{array}{l}\text { Knowledgeability and skills of the } \\
\text { Employees }\end{array}$ & $\begin{array}{l}\text { Very knowledgeable, } \\
\text { polite and friendly }\end{array}$ & $\begin{array}{l}\text { Not very knowledgeable, } \\
\text { polite and friendly }\end{array}$ \\
\hline Unconditional service guarantee offered? & No & Yes \\
\hline
\end{tabular}

Table III. A sample choice set (note: each customer and each manager responded to 32 such choice sets.

\begin{tabular}{lc}
\hline Attributes & Estimated model \\
\hline Price of a large pizza & $-0.146^{*}$ \\
Waiting time (to seat) & $-0.041^{*}$ \\
Waiting time (to order) & $-0.025^{*}$ \\
Waiting time (after ordering) & $-0.039^{*}$ \\
Types of pizza crust available & $0.144^{*}$ \\
Types of pizza toppings available & $0.109^{*}$ \\
Amount of toppings on the pizza & $0.261^{*}$ \\
Amount of cheese on the pizza & $0.206^{*}$ \\
Pizza crust uniformity & $0.086^{*}$ \\
Pizza taste consistency & $0.122^{*}$ \\
Aesthetics of the restaurant & $0.067^{*}$ \\
Reliability of service & $0.266^{*}$ \\
Will handle unique customer demands? & $0.154^{*}$ \\
Knowledgeability and skills of the employees & $0.255^{*}$ \\
Unconditional service guarantee offered? & $0.111^{*}$ \\
Intercept & 0.064 \\
Statistical information & \\
Log-likelihood ratio & \\
McFadden's $\rho^{2}$ & \\
Adjusted McFadden's $\rho^{2}$ & 0.74 \\
Note: $*$-value < 0.05 & 0.71
\end{tabular}

Table IV. Estimated multinomial logit model for customers (89 randomly selected customers responded to 32 choice set each. Therefore the model is based on 2,848 responses)

the asymptotic $\mathrm{t}$-statistic for the $\beta$ parameters. As shown in Table IV, $\beta$ parameters for all 15 attributes were found to be statistically significant at the 5 per cent level.

Managers' perceptions of customer preferences

We contacted the six managers of two dine-in pizza restaurants, both of which were 
owned by the same corporation. University students are the majority of the customers of the three restaurants. We asked the managers to predict the choice patterns of their customers. We showed them the same 32 choice sets (presented to 89 customers of their company) and asked them if their student customers would choose "Restaurant \#1," "Restaurant \#2," or "Neither Restaurant." Then we compared the MNL model for customer choice to the MNL model for the managers' predictions of customer choice.

Table $\mathrm{V}$ presents the MNL logit model for the managers' perceptions of customer choice patterns. The log-likelihood ratio was 99.19 which is $\chi^{2}$ distributed with 16 degrees of freedom and is statistically significant at the 5 per cent level. The McFadden's $\rho^{2}$ was 0.42 even though the MNL model is based on data collected from only six respondents (192 responses). As shown in Table $\mathrm{V}, \beta$ parameters for seven out of 15 variables were found to be statistically significant at the 5 per cent level.

The MNL models contain a Gumbel scale parameter, which cannot be estimated separately from $\beta$. Therefore the $\beta$ parameters of two MNL logit models (Table IV and Table V) cannot be compared directly. Recently Swait and Louviere (1993) developed a procedure for comparing two MNL models which first estimates the relative $\mu$ for the two models and then re-scales the $\beta$ parameters of one MNL model in terms of the second model. Next a $\chi^{2}$ test is conducted to compare the re-scaled MNL model to the second model. We conducted similar tests for the MNL models presented in Table IV and Table V. Table V shows the re-scaled

\begin{tabular}{|c|c|c|}
\hline Variable & $\begin{array}{l}\text { Rescaled customer } \\
\text { model (\#) }\end{array}$ & Manager model \\
\hline Price of a Large pizza & -0.1058 & -0.004 \\
\hline Waiting time (to seat) & -0.0297 & -0.004 \\
\hline Waiting time (to order) & -0.0181 & $-0.033 *$ \\
\hline Waiting time (after ordering) & -0.0283 & -0.006 \\
\hline Types of pizza crust available & 0.1044 & 0.047 \\
\hline Types of pizza toppings available & 0.0790 & $0.278^{*}$ \\
\hline Amount of toppings on the pizza & 0.1892 & $0.420^{*}$ \\
\hline Amount of cheese on the pizza & 0.1493 & $0.331 *$ \\
\hline Pizza crust Uniformity & 0.0623 & -0.520 \\
\hline Pizza taste consistency & 0.0884 & 0.141 \\
\hline Aesthetics of the restaurant & 0.0486 & 0.161 \\
\hline Reliability of service & 0.1928 & $0.290 *$ \\
\hline $\begin{array}{l}\text { Will handle unique customer } \\
\text { demands? }\end{array}$ & 0.1116 & $0.232 *$ \\
\hline $\begin{array}{l}\text { Knowledgeability and skills of the } \\
\text { employees }\end{array}$ & 0.1848 & $0.456^{*}$ \\
\hline $\begin{array}{l}\text { Unconditional service guarantee } \\
\text { offered? }\end{array}$ & 0.0805 & 0.150 \\
\hline Intercept & 0.0464 & $1.287 *$ \\
\hline $\begin{array}{l}\text { Statistical information } \\
\text { Log-likelihood ratio } \\
\text { McFadden's } \chi^{2} \\
\text { Adjusted McFadden's } \chi^{2}\end{array}$ & & $\begin{array}{c}\left.99.19 \text { ( } \chi^{2} \text { with } 16 \text { d.f. }\right)^{*} \\
0.42 \\
0.27\end{array}$ \\
\hline \multicolumn{3}{|c|}{$\begin{array}{l}\text { Notes: } * p \text {-value }<0.05 \\
\text { \#NtELOGIt found Optimum relative } \mu \text { for Customer-Manager Model pair to be } 1.3795 \text {. then } \\
\text { the customer model (Table IV) was re-scaled and a } \chi^{2} \text { test for the equality of parameters } \\
\text { between the re-scaled customer model and the manager model was conducted. Table V } \\
\text { contains the re-scaled customer model for the sake of visual comparison. the original } \beta \\
\text { parameters for the customer model were divided by the relative } \mu \text { to obtain the re-scaled } \\
\text { customer model. the } \chi^{2} \text { test conducted by NtELOGIT showed that the } \beta \text { parameters for the } \\
\text { customer and manager MNL models were not the same (at } \mathrm{p}<0.0000 \text { ). }\end{array}$} \\
\hline
\end{tabular}

Table V. Managers' perceptions of customer choice patterns (six managers responded to 32 choice sets, each; therefore the model is based on 192 responses) 
customer choice model adjacent to the model of managers' predictions of customer choice. A simple visual comparison shows that the two sets of $\beta$ parameters are not the same. The $\chi^{2}$ test was conducted by NTELOGIT, which showed that the $\beta$ parameters for the two MNL models are not the same. In other words, we found that the managers' perceptions of customer choice patterns are not the same as the customers' actual choice patterns.

\section{Managerial Implications}

Several articles and books emphasize the need for customer-based operations management in service industries. In this article we have presented an effective approach for positioning service operations based on customer tastes and preferences. DCA can be used to identify relative weights for service attributes from the customers' points of view. The discretechoice weights show how changing a particular attribute level affects market share (and therefore profitability). As mentioned earlier the MNL model presented in Tables IV, and 5 can be easily incorporated into a spreadsheet as a decision support system and the managers can use this model to evaluate the change in market share if one or more attributes are changed by them or their competitors.

For an actual application, it is important to validate that the experimental subjects are true representatives of the population of interest. However, for the sake of the example, we will assume that the customer choice result presented in Table IV can be generalized to all the customers of the pizza restaurants in the geographical area. We also assume that there are only three pizza restaurants (Shop \#1, Shop \#2, Shop \#3) in the geographical region from which the data was collected. All three restaurants are dine-in facilities but the attribute levels are different for the three restaurants. Table $\mathrm{VI}$ shows the design codes $( \pm 1,+1$ or 0$)$ for these restaurants. The \pm 1 and +1 values represent the dine-in pizza restaurant attributes levels specified in Table II. For example, a " \pm 1 " for the "Aesthetics of the restaurant" indicates that the restaurant looks like a fast-food establishment and a " +1 " indicates that it looks like an Italian restaurant. A design code of " 0 " represents the average value between \pm 1 and +1 . For example, a "0" for "types of pizza available" represents "two types of crust."

Most of the attribute levels for the three pizza restaurants presented in Table VI are different from each other. Therefore the utility of each restaurant for customers should be expected to be different from each other. The MNL model developed for the customers (Table IV) can be used to calculate the utility for each of the three pizza restaurants and their expected market share (based on the assumption that the MNL model represents the choice patterns of all customers in the region). As shown in Table VI, the attribute levels for the pizza restaurants are multiplied by the corresponding $\beta$ value and then summed to get an overall utility for each restaurant. Next the MNL model provides the expected market share for each restaurant. As shown in Table VI, Shop \#1, Shop \#2 and Shop \#4 are expected to have 44.96 per cent, 28.61 per cent and 26.44 per cent market shares, respectively. 


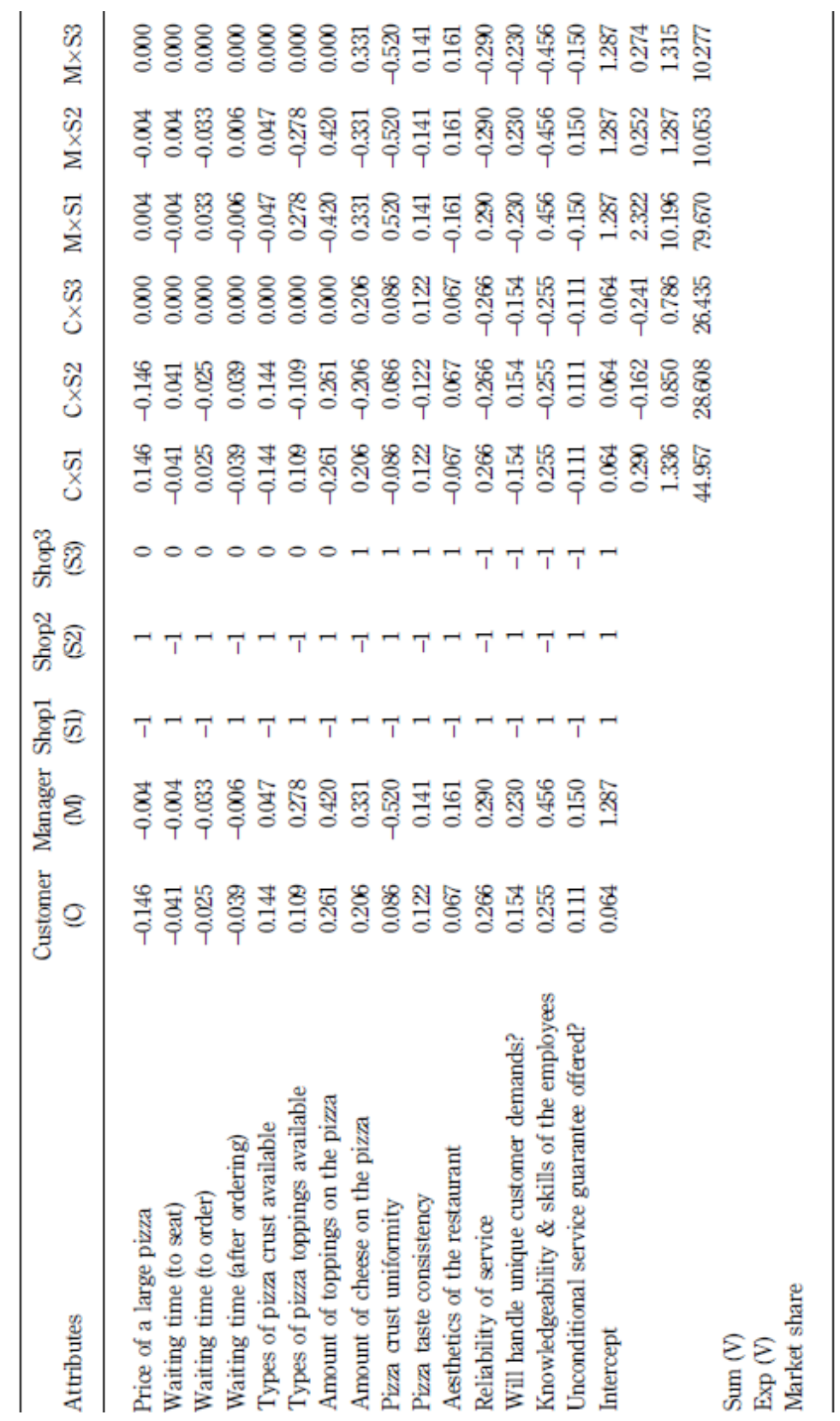

Table VI. Spreadsheet-based decision support model for managerial "what-if" analysis

Table $\mathrm{VI}$ also shows the expected market share for the three pizza restaurants based on managers' perceptions. Based on managers' perceptions of customer preferences, Shops \#1, \#2 and \#3 should have market shares of 79.67 per cent, 10.05 and 10.28 per cent respectively. In other words, the simple analysis presented in Table VI shows that the pizza companies will make extremely poor decisions regarding the structure of their operations if the gap between the customers' preferences and managers' perceptions are not eliminated. Such analyses further enhance the value of DCA for service managers.

The decision support system presented in Table VI directly links a firm's market 
performance to its operating priorities. For example, using the decision support system a manager can predict any changes in market share following a change in one or more attribute levels. The cost of changing any attribute level (for example, reduction in waiting time by increasing capacity or hiring more workers) can be compared to the revenue generated by expected market share gained before implementing the change in any attribute of the operating system. For example, assume that Shop \#1 increases its price of large pizza from \$11 to $\$ 16$ (from design code \pm 1 to design code +1 ). Recalculating the market shares for the three restaurants (using equation (1) and (2)) yields a new market share of 37.88 per cent for Shop \#1. In other words, Shop \#1 will lose approximately 7 per cent market share by making such a move. If we assume that the MNL model represented in Table V was for the managers of Shop $\# 1$ and recalculate the market share for the three shops using their perceptions, we do not see any major changes in the expected market share of Shop \#1. In other words, the manager's model underestimates the impact of a price change on customer demand patterns. Should a decision to increase price be made, based on the managers' model, the firm might seriously hurt itself in the marketplace. Similar analyses can be conducted for other attributes (waiting times, service guarantee, etc.).

The decision-support system is general is nature and therefore allows one to estimate the effect of multiple changes in attribute levels. By way of example, assume that Shop \#1 changes its price to $\$ 13.50$ (a design code of 0 ) and amount of topping from little to lots (a design code of +1 ); that Shop \#2 changes the amount of toppings from lots to little (a design code of 1) and amount of cheese from little to lots (a design code of +1 ); and that Shop \#3 changes its price to $\$ 11$ (a design code of \pm 1 ) and changes types of crust to one (a design code of \pm 1 ). The new expected market shares will be then 55.68 per cent, 21.79 per cent, 22.53 per cent respectively, for Shops \#1, \#2, and \#3. The corresponding market share predictions based on the managers' perceptions are 90.96 per cent, 4.16 per cent and 4.87 per cent.

\section{Conclusions}

This study has presented an approach for positioning services' operational priorities (quality, cost, delivery, and flexibility) based on customer preferences. We have demonstrated how discrete choice experiments can be designed and conducted in service operations. We also show that managers' perceptions of customer preferences might not accurately predict how customers choose services in the marketplace. Since "customer contact" is an important aspect of service businesses, it is important for the managers to understand the needs of the customers (Chase, 1981; Schmenner, 1986). However, often the managers of the service businesses are far removed from the customers and so they might not understand customer needs exactly. Similarly, managers in different functional areas within one organization might differ in their perceptions of customer choice patterns. Understanding managers' perceptions of customer choice patterns will provide very valuable information, then, should management desire to have their operations deliver what customers want.

Using the MNL model developed for the customers, managers can position their 
operations to better meet market needs. Ideally managers would know exactly what their customers need and design and manage their service businesses accordingly. However, most companies do not operate under ideal conditions. For example, the results presented in Table $\mathrm{V}$ show that managers' perceptions of customer preferences are not the same as customers' actual preferences. Such information can be very valuable for quality and process improvement. Management can focus their attention on bridging the "gaps" between their perceptions and those of their customers and use the MNL models (Table IV and Table V) as a continuous improvement tool.

The discrete choice results presented in this paper can be used to conduct numerous such decision-making analyses. Once a MNL model has been set up it can be easily used as a decision support system for evaluating, from the customers' points of view, any changes in the design of new product-service packages and/or changing the attributes of existing offerings.

The objective of this study was to show how one could use DCA to effectively integrate customer preferences and choices into operating decisions in services. We have presented the methodology and an empirical example from the pizza industry and have demonstrated the usefulness of DCA as a decision support tool. We feel that the use of customer-based approaches similar to the one presented here can significantly improve the financial and market positions of firms operating in competitive service industries.

\section{References}

Anderson, N.H. (1981), Foundations of Information Integration Theory, Academic Press, New York, NY.

Anderson, N.H (1982), Methods of Information Integration Theory, Academic Press, New York, NY.

Anderson, J.C., Cleveland, G. and Schroeder, R.G. (1989), "Operations strategy: a literature review", Journal of Operations Management, Vol. 8 No. 2, pp. 133-58.

Ben-Akiva, M., and Lerman, S.R. (1991), Discrete Choice Analysis, The MIT Press, Boston, MA.

Bishop, Y.M.M, Fienberg, S.E. and Holland, P.W. (1975), Discrete Multivariate Analysis: Theory and Practice, The MIT Press, Cambridge, MA.

Chase R.B. (1981), "The customer contact approach to services: theoretical bases and practical extensions", Operations Research, Vol. 29 No. 4.

Chase, R.B. (1990), "Where does the customer fit in a service operation", Harvard Business Review, November-December.

Chase, R.B. (1996), "The mall is my factory: reflections of a service junkie", Production and Operations Management, Vol. 5 No. 4, pp. 298-308. 
Davidow, W.H. and Uttal, B. (1989), "Service companies: focus or falter", Harvard Business Review, July-August.

Davis, M.M. (1991), "How long should a customer wait for service?" Decision Sciences, Vol. 22, pp. 421-34.

Flynn, B., Jakakibara, S., Schroeder, R.G., Bates, K.A. and Flynn, E.J. (1990), 'Empirical research methods in operations management", Journal of Operations Management, Vol. 9 No. 2, 1990, pp. 250-84.

Griffin, A. and Hauser, J.R. (1993), "The voice of the customer", Marketing Science, Vol. 12 No. 1, pp. 1-27.

Hagerty, M.R. (1986), "The cost of simplifying preference models", Marketing Sciences, Vol. 5 No. 4, pp. 298-319.

Hahn, G.J. and Shapiro, S.S. (1966), A Catalog and Computer Program for the Design and Analysis of Orthogonal Symmetric and Asymmetric Fractional Factorial Experiments, General Electric Research and Development Center, New York, NY.

Hart, C.W.L. (1988), "'The power of unconditional service guarantees", Harvard Business Review, July-August.

Hayes, R.H. and Wheelwright, S.C. (1984), Restoring Our Competitive Edge: Competing Through Manufacturing, John Wiley \& Sons, New York, NY.

Heskett, J.L. (1987), "Lessons in the service sector", Harvard Business Review, March-April.

Intelligent Marketing Systems (1992), CONSERV, Software Program and User's Manual, Edmonton.

Intelligent Marketing Systems (1992), NTELOGIT, Software Program and User's Manual, Edmonton.

Kellog, D.L. and Nie, W. (1995), "A framework for strategic service management", Journal of Operations Management.

Lindsley, W.B., Blackburn, J.D. and Elrod, T. (1991), "'Time and product variety competition in the book distribution industry", Journal of Operations Management, Vol. 10 No. 3, pp. 344-62.

Louviere, J.J. (1988), Analyzing Decision Making: Metric Conjoint Analysis, Sage Publications, Newbury Park, CA.

Louviere, J.J and Timmermans, H. (1990), "Stated preferences and choice models applied to recreation research: a review", Leisure Sciences, Vol. 12, pp. 9-32. 
Louviere, J.J and Timmermans, H. (1990), “A review of recent advances in decompositional preference and choice models", Journal of Economic and Social Geography, Vol. 81 No. 3, pp. 214-24.

Louviere, J.J., Oppewal, H., Timmermans, H. and Thomas, T. (1995), “Handling large number of attributes in conjoint analysis: who says existing techniques can't be applied? But if you want an alternative, how about hierarchical choice experiments?", working paper, David Eccles School of Business, University of Utah, Provo, UT.

Lovelock, C.H. (1983), "Classifying services to gain strategic marketing insights", Journal of Marketing, Vol. 47, pp. 9-20.

Lovelock, C.H. (1992), "A basic toolkit for service management", in Managing Services: Marketing, Operations, and Human Resources, 2nd ed., Prentice-Hall, Englewood Cliffs, NJ.

McFadden, D. (1986), "The choice theory approach to marketing research", Marketing Science, Vol. 5 No. 4, pp. 275-97.

McLean, R. and Anderson, V. (1984), Applied Factorial and Fractional Designs, Marcel Deckker, New York, NY.

Meredith, J.R., Raturi, A., Amoako-Gyampah, K. and Kaplan, B., (1989), 'Alternative research paradigms in operations management", Journal of Operations Management, Vol. 8 No. 4, pp. 297-326.

Mersha, T. (1990), "Enhancing the customer contact model", Journal of Operations Management, Vol. 9 No. 3, pp. 391-405.

Schmenner, R.W. (1986), "How can service businesses survive and prosper", Sloan Management Review, Vol. 27 No. 3, pp. 21-32.

Swait, J. and Louviere, J.J. (1993), "The role of the scale parameter in the estimation and comparision of multinomial logit models", Journal of Marketing Research, Vol. 30, pp. 305-14.

Swamidass, P.M. (1991), "Empirical science: new frontier in operations management research", Academy of Management Review, Vol. 16 No. 4, pp. 793-814.

Verma, R. and Thompson, G.M. (1996), "'Basing service management on customer determinants: the importance of hot pizza", Cornell Hotel and Restaurant Administration Quarterly, Vol. 37 No. 3, pp. 18-23.

Verma, R., Thompson, G.M. and Louviere, J.J. (1999), "'Discrete choice analysis in hospitality management research", Journal of Service Research, 1999, Vol. 1 No. 3 (forthcoming). 
Wemmerlov, U. (1990), "A taxonomy for service processes and its implications for system design", International Journal of Service Industry Management, Vol. 1 No. 3, pp. 13-27. 